\title{
Editorial
}

\section{Computational Intelligence in Image Processing 2018}

\author{
Erik Cuevas $\mathbb{D}^{1},{ }^{1}$ Daniel Zaldívar $\mathbb{D}^{1},{ }^{1}$ Gonzalo Pajares $\mathbb{D}^{2}{ }^{2}$ \\ Marco Perez-Cisneros $\mathbb{1}{ }^{1},{ }^{1}$ and Raúl Rojas ${ }^{3}$ \\ ${ }^{1}$ Departamento de Electrónica, CUCEI, Universidad de Guadalajara, Avenida Revolución 1500, Guadalajara, JAL, Mexico \\ ${ }^{2}$ Departamento de Ingeniería de Software e Inteligencia Artificial, Facultad Informática, Universidad Complutense de Madrid, \\ 28040 Madrid, Spain \\ ${ }^{3}$ Institut für Informatik, Freie Universität Berlin, Arnimallee 7, 14195 Berlin, Germany \\ Correspondence should be addressed to Erik Cuevas; erik.cuevas@cucei.udg.mx
}

Received 27 February 2018; Accepted 27 February 2018; Published 5 August 2018

Copyright (C) 2018 Erik Cuevas et al. This is an open access article distributed under the Creative Commons Attribution License, which permits unrestricted use, distribution, and reproduction in any medium, provided the original work is properly cited.

Computational intelligence (CI) represents a set of robust information processing approaches for knowledge management and decision-making. CI methods are considered as useful tools for the development of advanced systems which maintain intelligent capabilities such as learning, adaptation, and evolution for solving complex problems. Examples of popular CI schemes include artificial neural networks, fuzzy systems, evolutionary algorithms, decision trees, multiagent systems, knowledge-based systems, rough set theory, and hybridization of these models.

Image processing is a progressive and fast-moving research discipline. Recent advances in image processing have produced an explosion in the use of images in a diversity of engineering and scientific applications. Therefore, each new approach that is developed by engineers, mathematicians, and computer scientists is quickly identified, understood, and assimilated in order to be applied to image processing problems.

Standard image processing techniques frequently face great difficulties when they operate over images containing information that is incomplete, noisy, imprecise, fragmentary, not fully reliable, vague, contradictory, deficient, and overloading. Under such conditions, the use of computational intelligence approaches has been recently extended to address challenging real-world image processing problems.

The use of computational intelligence approaches in image processing has increased in all engineering areas. Such a fact is evident from a quick look at special issues, congresses, and specialized journals that focus on such a topic. The central purpose of this special issue is to bridge the gap between computational intelligence techniques and challenging image processing applications. The final goal is to expose the cutting-edge research and applications that are going on across the domain of image processing, particularly those whose contemporary computational intelligence techniques can be or have been successfully employed.

The special issue received several high-quality submissions from different countries all over the world. All submitted papers have followed the same standard of peer-reviewing by at least three independent reviewers, just as it is applied to regular submissions to the Mathematical Problems in Engineering journal. The primary guideline has been to demonstrate the wide scope of computational intelligence algorithms and their applications to image processing problems.

The paper authored by S. Lee and I. Kim presents a deep neural network model for effective video captioning. Apart from visual features, the proposed model learns additionally semantic features that describe the video content effectively. In this model, visual features of the input video are extracted using convolutional neural networks such as C3D and ResNet, while semantic features are obtained using recurrent neural networks such as LSTM. The approach also includes an attention-based caption generation network to generate the correct natural language captions based on the multimodal video feature sequences. Various experiments, conducted with the two large benchmark datasets, Microsoft Video Description (MSVD) and Microsoft Research VideoTo-Text (MSR-VTT), demonstrate the performance of the proposed model. 
X. Liu and X. Gao propose a method based on the subspace optimization to improve the image restoration process. This method corrects the search directions of primal alternating direction method by using the energy function and a linear combination of the previous search directions. In addition, the convergence of the primal alternating direction method is proven under some weaker conditions. Thus, the convergence of the corrected method could be easily obtained by the equivalence between the direction method and the previous direction. Numerical examples are given to show the performance of the proposed method finally.

C. Jungan et al. propose a novel clustering algorithm called $k$-deviation density based DBSCAN (kDDBSCAN). The method extends the DBSCAN method by exploiting a new density definition. Various datasets containing clusters with arbitrary shapes and different densities are used to demonstrate its performance and investigate its feasibility. The results show that kDDBSCAN performs better than DBSCAN.

O. O. Olugbara et al. present a new algorithm based on perceptual color difference saliency along with binary morphological analysis for segmentation of melanoma skin lesion in dermoscopic images. The new algorithm is compared with existing image segmentation algorithms on benchmark dermoscopic images acquired from public corpora. Results of both qualitative and quantitative evaluations of the new algorithm are encouraging as the algorithm performs excellently in comparison with the existing image segmentation algorithms.

The paper by A. Stubendek and K. Karacs proposes a combined shape descriptor for object recognition with offline and online learning methods. The descriptor is composed of a local edge based part and global statistical features. The approach also presents a two-level, nearest neighborhood type multiclass classification method, in which classes are bounded defining an inherent reject region. In the first stage, built on the global features, class candidates get prefiltered, in contrast to the second stage, where the projected features are compared. The experimental results show that the combination of independent features leads to increased recognition robustness and speed. The core algorithms map easily to cellular architectures or dedicated VLSI hardware.

$\mathrm{H}$. Xu et al. present a study on crack detection of industrial explosives. The proposed algorithm consists of the following steps: (1) Image preprocessing was performed according to the defect features of industrial explosives cartridge, and we developed an improved visual attention-based algorithm. This proposed algorithm features a parametric analysis that can be implemented on the image according to the conspicuous maps with the introduction of the concept of defect discrimination. (2) As compared with other algorithms, our method can realize real-time multitarget detection function. (3) A new analysis method, the IPV-WEN algorithm, was proposed to analyze the cartridge defects based on performance indices. Through comparison and experimentation, it was revealed that this method can achieve a detection accuracy of $97.9 \%$, with detection time of $34.51 \mathrm{~ms}$, which satisfied the requirement in the industrial explosives production.
The paper authored by W. Zhao et al. presents a denoising algorithm combined with SGK dictionary learning and the principal component analysis (PCA) for noise estimation. At first, the noise standard deviation of the image is estimated by using the PCA noise estimation algorithm. And then it is used for SGK dictionary learning algorithm. Experiments' results show that (1) the SGK algorithm has the best denoising performance compared with the other three dictionary learning algorithms, (2) the SGK algorithm combined with PCA is superior to the SGK combined with other noise estimation algorithms, and (3) compared with original SGK algorithm, the proposed algorithm has higher PSNR and better denoising performance.

M. Yousaf et al. propose an effective novel technique to improve the performance of content-based image retrieval (CBIR) on the basis of visual words fusion of scale-invariant feature transform (SIFT) and local intensity order pattern (LIOP) descriptors. SIFT performs better on scale changes and on invariant rotations. However, SIFT does not perform better in the case of low contrast and illumination changes within an image, while LIOP performs better in such circumstances. SIFT performs better even at large rotation and scale changes, while LIOP does not perform well in such circumstances. Moreover, SIFT features are invariant to slight distortion as compared to LIOP. The proposed technique is based on the visual words fusion of SIFT and LIOP descriptors, which overcomes the aforementioned issues and significantly improves the performance of CBIR. The experimental results of the proposed technique are compared with another proposed novel features fusion technique based on SIFT-LIOP descriptors as well as with state-of-the-art CBIR techniques. The qualitative and quantitative analyses carried out on three image collections, namely, Corel-A, Corel-B, and Caltech-256, demonstrate the robustness of the proposed technique based on visual words fusion as compared to features fusion and state-of-the-art CBIR techniques.

S. Cheng et al. present a method for water-quality monitoring. The approach is based on the Tracking-LearningDetection (TLD) framework and eXtreme Gradient Boosting (XGBoost). Firstly, TLD captures 3D coordinate information of fish by using video. Then, it calculates the parameters of the fish movement which can reflect the change of water quality through the processing of the fish body information. The data coordinate information will be more prominent via the data processing. The model was used to analyze and evaluate fish behavior parameters under unknown quality to achieve the purpose of water-quality monitoring.

The paper authored by P. V. V. Kishore et al. presents an approach to detect Indian classical dance forms. With this propose, a new segmentation model is developed using discrete wavelet transform and local binary pattern (LBP) features. Then, a 2D point cloud is created to detect local human shape changes in subsequent video frames. The classifier is fed with 5 types of features calculated from Zernike moments, $\mathrm{Hu}$ moments, shape signature, LBP features, and Haar features. The method also explores multiple feature fusion models with early fusion during segmentation stage and late fusion after segmentation for improving the classification process. The extracted features input the AdaBoost multiclass classifier 
with labels from the corresponding song (tala). The classifier has been tested with online dance videos and on an Indian classical dance dataset prepared in our lab. The algorithms were tested for accuracy and correctness in identifying the dance postures.

E. Chavolla et al. propose an algorithm based on a modification of the HSV color model in order to improve the accuracy of the results obtained from the color segmentation by using the $K$-means++ algorithm. The proposal gives a better segmentation and fewer erroneous color detections due to illumination conditions. This is attained by shifting the hue and rearranging the $H$ equation in order to avoid undefined conditions and increase robustness in the color model.

\section{Acknowledgments}

Finally, we would like to express our gratitude to all of the authors for their contributions and the reviewers for their efforts to provide valuable comments and feedback. We hope this special issue offers a comprehensive and timely view of the area of applications of computational intelligence in image processing and we hope that it will grant stimulation for further research.

Erik Cuevas Daniel Zaldívar Gonzalo Pajares Marco Perez-Cisneros Raúl Rojas 


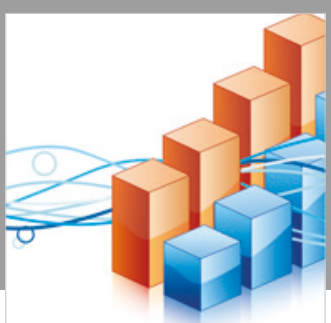

Advances in

Operations Research

\section{-n-m}
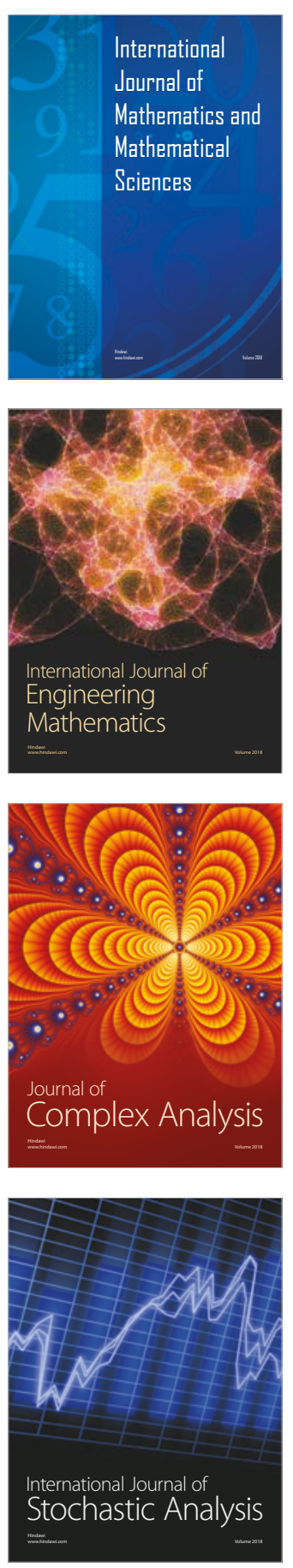
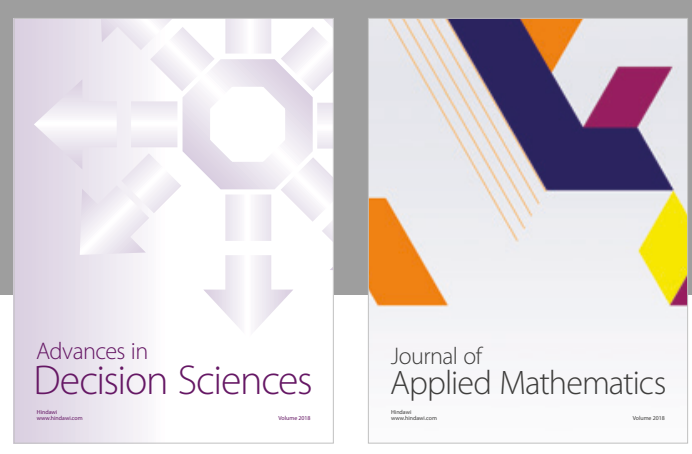

Journal of

Applied Mathematics
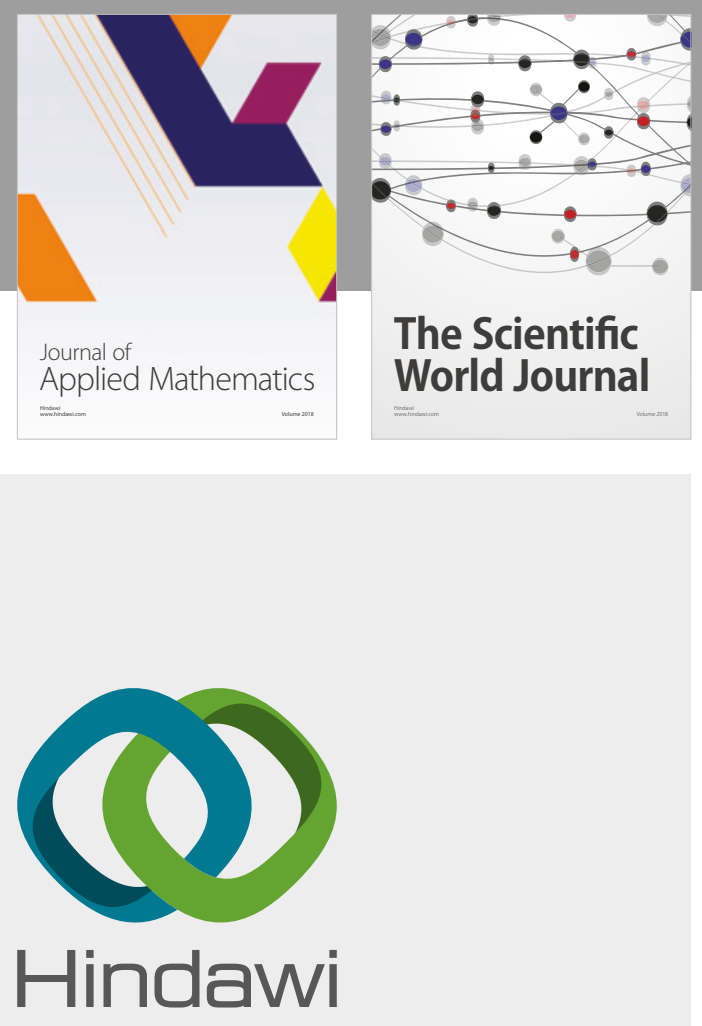

Submit your manuscripts at

www.hindawi.com

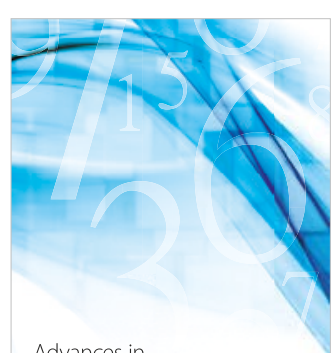

Advances in
Numerical Analysis
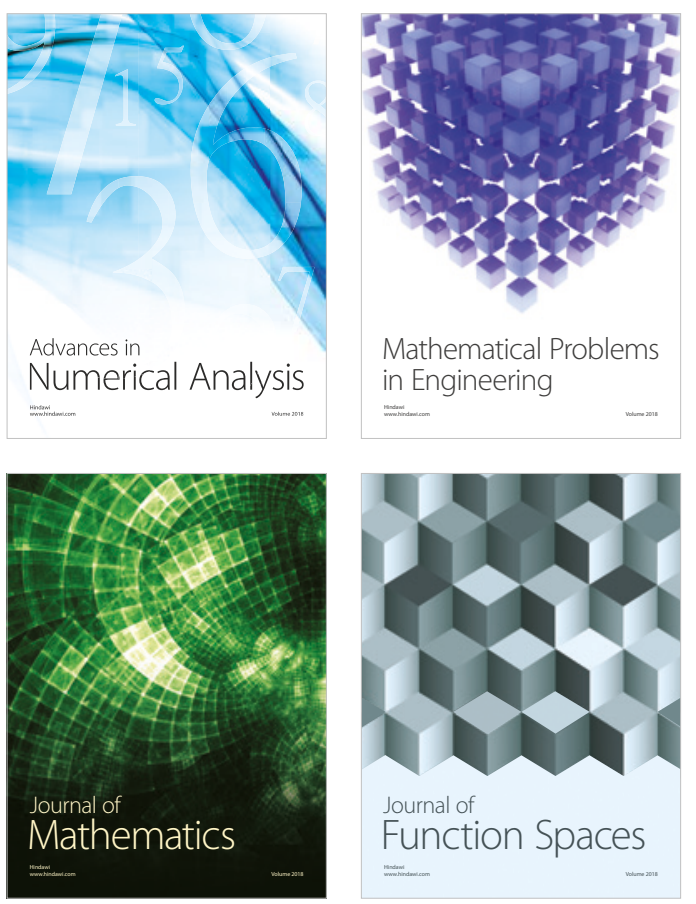

Mathematical Problems in Engineering

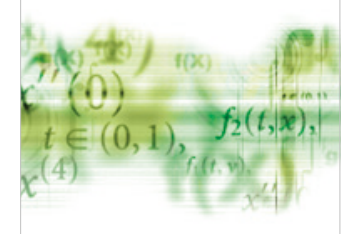

International Journal of

Differential Equations

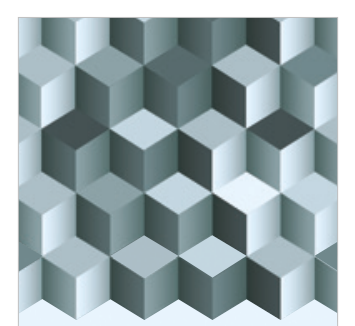

Journal of

Function Spaces

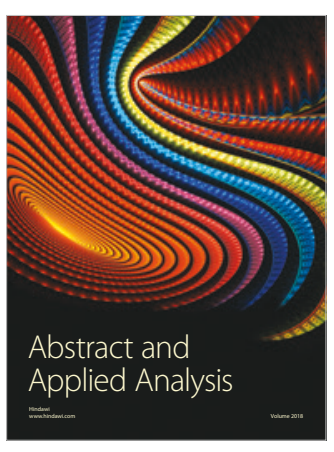

The Scientific

World Journal

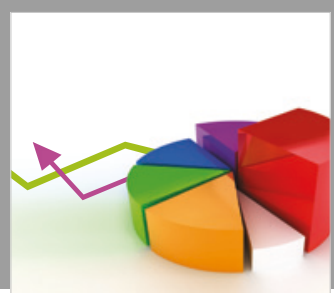

Journal of

Probability and Statistics
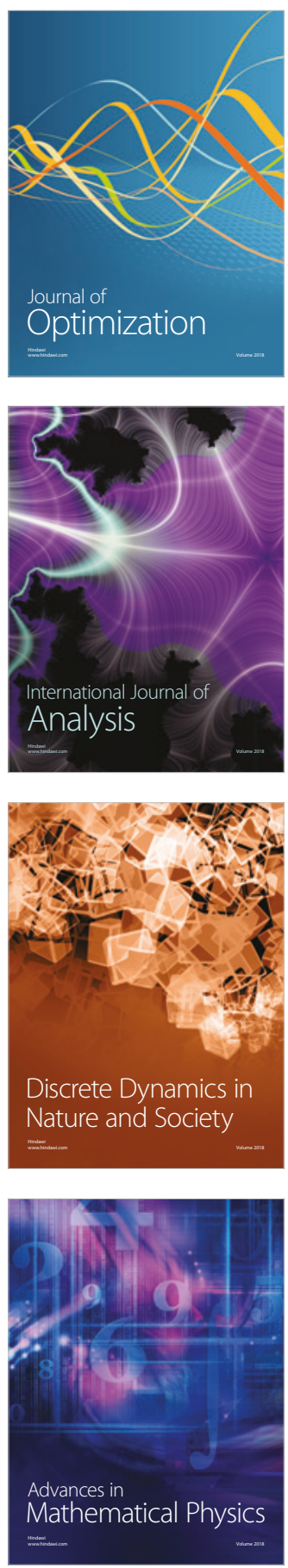